\title{
Formación educativa y desarrollo profesional. El caso de egresadas(os) de especialidades de posgrado en enfermería*
}

\section{Educational formation and professional development. For graduates of Unique Nursing Specialization Plan}

Mtra. Reyna Matus Miranda. Dra. Lasty Balseiro Almario.• Mtra. Rosa A. Zárate Grajales• Mtra. Mercedes García Cardona...

Lic. Cristina Balan Gleaves....

\section{Resumen}

Objetivo. Analizar, a partir de la percepción de los egresados del Plan Único de Especialización en Enfermería (PUEE), en que medida los estudios realizados han contribuido a su desarrollo profesional. Metodología: Se diseñó un instrumento tipo escala de Likert para evaluar la percepción del desarrollo profesional alcanzado, éste se sometió a validación de contenido, quedó integrado por 24 reactivos correspondientes a cuatro dimensiones: Responsabilidad, Autonomía, Trabajo en equipo y Posicionamiento. Se consideraron además características generales de los egresados y de las instituciones donde se labora; la muestra la constituyeron 417 especialistas de un universo de 1067 egresados del PUEE entre 1997-2007; para el tratamiento de la información se utilizó estadística descriptiva e inferencial. Resultados: Respecto a la inserción laboral la mayoría de los egresados trabaja en Instituciones públicas de 3er nivel de atención, de acuerdo a su percepción tienen remuneraciones acordes al puesto y realizan actividades relacionadas en forma directa con el cuidado; se observó relación entre haber obtenido el grado de especialista y tener la plaza correspondiente o una categoría de mayor responsabilidad; los egresados de las especialidades con mejor posicionamiento son los de Enfermería Perinatal, Cardiovascular e Infantil. Discusión: La inserción laboral de los egresados del PUEE es acorde a las expectativas del Plan de Estudios. De acuerdo a la percepción de los egresados la formación académica recibida les brinda habilidades para actuar con responsabilidad, autonomía y trabajo en equipo, sin embargo no les ha permitido en igual medida obtener reconocimiento institucional, de colegas o mejorar su posición laboral.

* Este artículo se deriva del Proyecto PAPIIT IN-30841.

- Profesoras de Carrera TC Asociado C ENEO-UNAM.

..Profesora de Carrera TC Titular B ENEO-UNAM.

...Profesora de Asignatura A ENEO-UNAM.

....Profesora de Asignatura B ENEO-UNAM.

FECHA DE RECIBIDO: 6 DE JULIO 2011

FECHA DE ENVIADO: 10 DE JULIO 2011

FECHA DE ACEPTADO: 20 DE SEPTIEMBRE DE 2011 


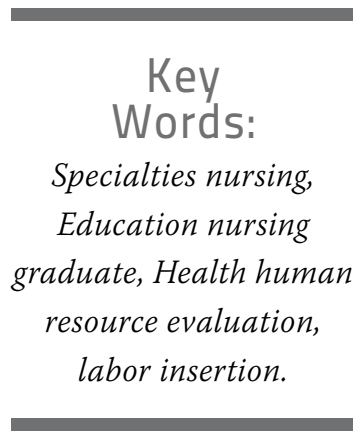

\begin{abstract}
Objective: considering the perception of graduates from the Unique Nursing Specialization Plan (UNSP), analyze the contribution extent of this program to their professional development. Methodology: a Likert-scale instrument was designed to assess the perceived professional development. The content validated questionnaire included 24 items related to four dimensions: Responsibility, Autonomy, Teamwork, and Working-Positioning. General characteristics and working-place details were also gathered. A sample of 417/1067 graduates from the 1997-2007 generations was formed and descriptive and inferential statistics were used for the analysis. Results: most graduates work in 3rd level attention public institutions and, according to their perception, have position-proportional earnings and perform activities directly related to care. An association between having obtained a specialization degree and holding a corresponding working-position or higher responsibility activity was observed. The best placed specializations turned out to be newly born, cardiovascular, and children nursing. Discussion: labor-insertion of UNSP's graduates corresponds to the program expectations, and according to their perception, this academic formation gives them responsibility, autonomy and teamwork skills, but they feel they can receive more institutional and colleagues acknowledgement and thus manage better working-positions.
\end{abstract}

\section{INTRODUCCIÓN}

Existe en los últimos años un amplio consenso entre los estudiosos y responsables de la formulación y ejecución de políticas sociales en que la educación es uno de los mecanismos más eficaces con los que cuentan las Naciones para promover la equidad y la democracia. ${ }^{1,2}$ De manera general, se parte del supuesto que el aumento en los niveles educativos de la población contribuye a promover una distribución del ingreso económico más equilibrada y a combatir la pobreza. Esto se logra, debido a que la educación formal - aquella que está establecida dentro de un sistema educativo institucionalizado, cronológicamente graduado y jerárquicamente estructurado, que abarca desde la escuela primaria hasta los grados superiores universitarios ${ }^{3}$ - es considerada como uno de los principales mecanismos de movilidad social ascendente. ${ }^{4}$
Lo anterior sin perder de vista que el espacio escolar no es el único lugar de formación, de aprendizaje y de cultura ya que el fenómeno educativo ocurre en otros espacios y tiempos sociales: las empresas, las calles, las interacciones sociales, el trabajo e incluso, durante los momentos de ocio o descanso; como afirma Boff, no sólo se aprende con el cerebro ni en la escuela. Se aprende de la vida entera y en todas las formas de vivir. ${ }^{5}$

Partiendo de lo señalado, se puede concebir a la educación como un proceso social, continuo y permanente que se da a lo largo de toda la vida y en todos los lugares, debiendo ser desarrollada de forma participativa, es decir, de manera consciente y reflexiva acorde a la realidad que vive y enfrenta cada persona. Esto permite afirmar que la formación se recibe no sólo en la academia sino a lo largo de la vida, incluyendo los espacios laborales donde se asumen actividades y responsabilidades que van tomando un lugar determinante en la vida del ser humano, ayudándolo al desarrollo de sus capacidades y potencialidades.

Para el caso específico de la enfermería, a lo largo de su formación educativa de aproximadamente 5 años (nivel licenciatura), los alumnos reciben conocimientos y desarrollan habilidades que los preparan para prestar cuidados de enfermería de forma eficiente, segura y de calidad para que, vinculados a las necesidades del pais, participen (entre otras cosas) con una perspectiva crítica en la promoción de los cambios y transformaciones requeridos por la sociedad. ${ }^{6}$ Sin embargo, es imposible pretender que esta formación académica sea eficiente y adecuada para los treinta años de ejercicio 
laboral profesional que siguen a la conclusión de los estudios formales, particularmente si se considera que estamos inmersos en un proceso de mundialización y globalización ${ }^{7}$ que demanda profesionales autónomos y altamente calificados en el desarrollo de sus conocimientos, habilidades, destrezas, valores y actitudes que les permitan desempeñar adecuadamente un trabajo, una actividad o una tarea además de responder a los retos que representan las necesidades sociales y de salud en continuo cambio y/o evolución. Bajo este marco, es explicable la trascendencia y complejidad que adquiere la formación académica continua para y por el trabajo, donde por un lado, las Instituciones de salud emprenden acciones educativas a fin de estimular el desarrollo de capacidades de su personal de trabajo, para que respondan a las exigencias del avance tecnológico y por otro, desarrollar en ese profesional su vocación de crear, crecer, producir y obtener placer en su trabajo. ${ }^{8,9}$

Lo señalado cobra relevancia si se considera que el cuidado de enfermería exige una práctica enmarcada en una profunda responsabilidad y ética profesional, producto no sólo de una formación académica del mejor nivel obtenida en las aulas, sino de un propósito individual de búsqueda continua del conocimiento que justifique y respalde cada una de las acciones mediante las cuales enfermería cuida íntegramente la salud de las personas, ${ }^{10}$ sin perder de vista que también deberá dar respuesta a la necesidad de promoción profesional con el aumento correlativo en su responsabilidad y en los ingre- sos económicos percibidos.

Dentro de este contexto, las Instituciones de Educación Superior deben tomar un papel estratégico en la formación y en la actualización de los conocimientos y la formación integral del ser humano con una visión humanista y responsable ante las necesidades y oportunidades que le presente el entorno laboral, a fin de que éste se convierta en un ambiente de aprendizaje en el que haya lugar para la toma de decisiones y una mayor autonomía para actuar; bajo la perspectiva de Chiodelli, se trata deformar personas para pensar críticamente, resolver problemas, trabajar en equipo, para comprender la forma en que aprenden, de suerte que puedan aprender más. Es decir, deberán tener una educación que les permita afrontar situaciones ambiguas y difíciles en su vida o en su lugar de trabajo. ${ }^{11}$

Considerando lo anterior, este estudio aborda el caso específico de los Programas de Especialización de Posgrado que se desarrollan en la ENEO, mismos que tienen como base el Plan Único de Especialización en Enfermería (PUEE) el cual tiene como objetivo formar enfermeras especialistas en diversos campos de conocimiento, capaces de otorgar cuidados especializados de enfermería, teniendo como base un modelo de atención en el cual integren elementos teóricos, metodológicos e instrumentales, así como de las disciplinas de apoyo. ${ }^{12}$

A la fecha, a más de diez años de su implementación, este Plan que actualmente incluye trece especialidades, requiere ser evaluado en cuanto a sus logros en las siguientes vertientes: una para identificar en qué medida estos estudios po- sibilitaron el desarrollo profesional dentro y fuera de la propia institución de trabajo y otra, con relación al nivel de desempeño laboral teniendo como referencia el punto de vista de sus jefes inmediatos. Así, en esta primera etapa interesó conocer desde la perspectiva de los alumnos egresados del PUEE de las generaciones 1997-2008: ¿En qué medida los estudios de especialización en enfermería de posgrado contribuyeron a su desarrollo profesional?

\section{METODOLOGÍA}

Diseño y tipo de estudio; no experimental, transversal, descriptivo y comparativo.

Población y muestra: Se consideró incluir a todos los egresados de las especialidades de enfermería de posgrado de doce generaciones (1997 - 2007) que cumplieran con los siguientes criterios de inclusión; ser egresado de alguna de las especialidades de enfermería de posgrado de la ENEO de las generaciones consideradas, estar laborando en alguna institución o bien, en ejercicio independiente, que pudieran ser localizados por alguna de las vías establecidas para responder la encuesta y que aceptaran participar en el estudio posterior a darles a conocer los objetivos del mismo.

\section{Variable Independiente; Plan de Estudios de cada uno de los cursos de especialidad.}

El Plan de Estudios es un conjunto de experiencias de enseñanza/ aprendizaje realizadas dentro de cada especialidad a lo largo de dos semestres, que involucran un conjunto de contenidos seleccionados, para que al finalizar su formación sean capaces de otorgar cuidados de enfermería especializados a in- 


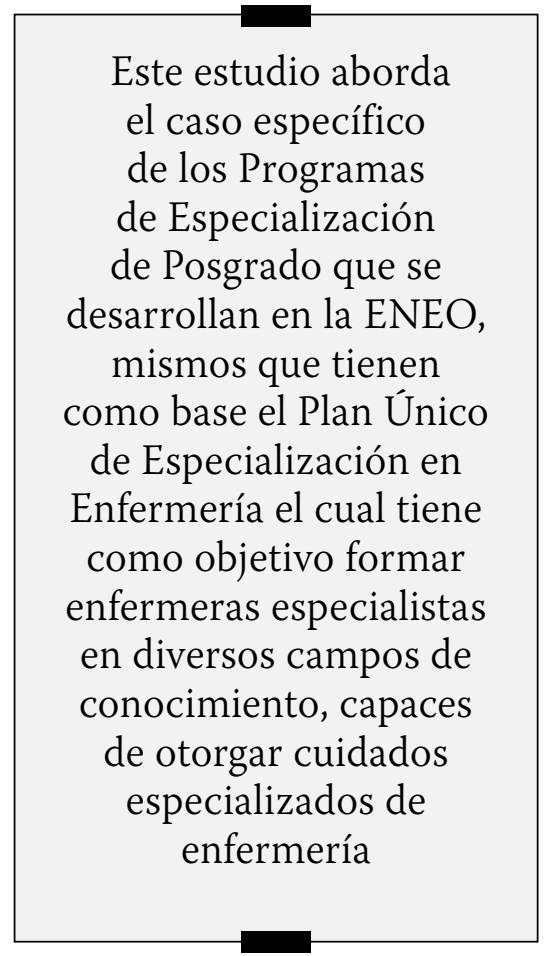

dividuos, teniendo como base un modelo de atención a través del cual integren elementos teóricos, metodológicos e instrumentales de la propia disciplina. ${ }^{13}$

\section{Variable Dependiente; Desarrollo Profesional.}

Se denomina Desarrollo Profesional al proceso intrínseco, continuo y dinámico que implica el despliegue de capacidades para tomar decisiones y ejecutar acciones independientes de enfermería asumiendo la responsabilidad de sus resultados. ${ }^{14}$ Va acompañado de un deseo de incrementar los conocimientos y habilidades a través del desarrollo académico en diversas modalidades en beneficio de la atención que se brinda. Comprende a su vez una actitud de disposición al trabajo colaborativo interdisciplinario y/o multidisciplinario en un ambiente de respeto. Lo anterior contribuirá a cambios laborales y en las percepciones económicas además del reconocimiento profesional y social.

\section{Dimensiones}

1.-Responsabilidad. Es el sentido ético de compromiso de carácter personal que implica el cumplimiento de las actividades y tareas inherentes al puesto profesional asumiendo las consecuencias. Se valora la contribución al alcance de los objetivos y metas institucionales así como la responsabilidad en el manejo adecuado de los recursos para proporcionar cuidados.

2.-Autonomía. Es la capacidad del egresado del PUEE de tomar decisiones propias y/o emprender acciones independientes dentro del ámbito de su competencia profesional, así como también el ejercicio independiente de la profesión. También de identificar sus necesidades o debilidades en el cuidado y buscar y/o emprender acciones académicas remediables como: asistencia a cursos, congresos, talleres, encuentros, búsquedas de evidencias para toma de decisiones etc.

3.-Trabajo en equipo. Es la disposición personal para la integración o vinculación con colegas u otros profesionales de la salud con el propósito de avanzar en el cuidado otorgado o en proyectos específicos, asumiendo incluso, en su caso, de manera libre o voluntaria el liderazgo (dirección) para la realización de tareas o actividades.

\section{4.-Posicionamiento. Son los} cambios profesionales (de categorías o puestos) los cuales van acompañados del incremento en sus percepciones salariales y/o condiciones laborales; alcanzando reconocimiento de colegas, otros profesionales o la sociedad en general dentro del ámbito laboral o social por la labor desempeñada, incluye la participación en alguna organización colegiada de enfermería.

\section{Instrumento de medición}

Con base en las variables y dimensiones consideradas, se procedió a la elaboración de un instrumento que permitiera aproximarnos a conocer el grado de desarrollo profesional de los egresados del PUEE desde su propia perspectiva.

La primera versión consideró tres secciones; la primera para identificar aspectos de ubicación laboral, estatus académico dentro del propio programa del PUEE. La segunda sección incluyó 40 enunciados que exploraban los indicadores de desarrollo profesional. Esta versión se sometió a una validación de contenido de expertos en el tema (dos investigadores, formados en el campo de la investigación educativa y dos enfermeras con amplia experiencia en la educación y la administración), quienes hicieron observaciones tanto a la primera sección, como a los enunciados incluidos en la segunda sección (redacción, número y características de los mismos). También sugirieron la inclusión de una tercera sección que explorará en qué medida en el último año habían realizado actividades consideradas de desarrollo profesional (pertenencia a grupos gremiales, búsqueda de evidencias relacionadas con su práctica). La segunda versión quedó con los ajustes sugeridos y fue 
con la que se aplicó una prueba piloto en una población parecida a la población blanco (20 enfermeras especialistas que laboran en una Institución de Seguridad Social).

Esta actividad permitió estimar el tiempo promedio de respuesta, así como la claridad de los enunciados y el aspecto de la conformación del instrumento en general. Derivado de esta aplicación y las sugerencias emitidas, se hicieron algunos ajustes en ciertos términos utilizados dentro del instrumento.

La aplicación a los egresados del PUEE de las generaciones consideradas, se ha realizado de diversas formas; al ser un instrumento autoadministrado, es factible su respuesta a través de la página web de la Escuela en el link establecido para tal fin, o bien en las propias coordinaciones del PUEE en las Unidades Sedes.

Una vez obtenida la información se creó una base de datos en SPSS 14.5 para concentrar y analizar la información.

De acuerdo a las características de las variables y las escalas de medición utilizadas (nominal, ordinal), se utilizó inicialmente estadística descriptiva y posteriormente inferencial; para la comparación global de las medias de los indicadores de los grupos se utilizó el análisis de varianza (ANOVA) y para la comparación de las medianas de cada uno de los indicadores al interior de cada grupo se uso la Prueba de Kruskal- Wallis y para hacer comparaciones específicas entre grupos se utilizó la t de Student para grupos independientes.

Para la realización de esta investigación nos adheriremos a los principios de la "Declaración de Helsinki" y a los de "La Ley Gene- ral de Salud", Título Segundo, de los Aspectos Éticos de la investigación en Seres Humanos. Capítulo I Disposiciones Comunes. Artículo 13 y 14.- En toda investigación en la que el ser humano sea sujeto de estudio, deberán prevalecer el criterio del respeto a su dignidad y la protección de sus derechos y bienestar. Esta investigación se considera de riesgo mínimo de acuerdo al artículo 17 y en cumplimiento con los aspectos mencionados en el Artículo 21.

No obstante, se solicitó el consentimiento informado a cada uno de los participantes y se les dio a conocer los objetivos y propósitos de la investigación.

\section{Resultados}

De las 1067 personas que han egresado entre 1997 a 2007 del Programa Único de Especialidades en Enfermería de la ENEO se lograron contactar y aceptaron contestar la encuesta 417, que constituyen el $39 \%$ del universo a encuestar. Es importante comentar que en algunos cuadros está información puede diferir ligeramente pues existen encuestas sin información en algunas variables.

Un primer aspecto de reflexión corresponde al entorno en el que surge este estudio; y tiene que ver

Ha habido incremento en el número de especialidades que se ofrecen, lo que obliga a evaluar en qué medida estos recursos humanos formados se insertan en el mercado de trabajo en cómo la ENEO da respuesta a las necesidades y requerimientos de atención especializada por parte de las instituciones del Sector Salud. Derivado de lo anterior, ha habido incremento en el número de especialidades que se ofrecen (actualmente son trece), lo que obliga a evaluar en qué medida estos recursos humanos formados se insertan en el mercado de trabajo, al ser la única institución que ofrece esta formación académica las (os) participantes mayoritariamente tienen su centro de trabajo en instituciones del D.F. y Estado de México.

Tradicionalmente en este ámbito profesional se distingue la participación predominante del sexo femenino, situación a la que no escapa este estudio, donde sólo uno de cada seis de los egresados es varón. Respecto a la edad poco más del $90 \%$ se encuentran ubicados dentro del rango de 24 a 44 años. Vale la pena señalar que este hecho se vincula con los años de antigüedad en el empleo pues poco más del $80 \%$ de la muestra se ubica en el rango de 15 años o menos de antigüedad.

En cada ámbito de trabajo existen situaciones que no pueden soslayarse; en este caso, como se sabe y se constata con los datos obtenidos hasta el momento, el profesional de enfermería, en un gran porcentaje, trabaja en una institución (pública o privada) por lo que necesariamente responde a la dinámica y necesidades existentes dentro de estos espacios y se ajusta y cumple los determinantes de carácter laboral; salarios, prestaciones, turnos, horarios, categorías, vacaciones, movimientos o cambios de categoría o escalafonarios establecidos en 
la forma de contratos de trabajo o condiciones generales de trabajo al cual se adhieren cuando ingresan y forman parte de una institución. Lo anterior puede observarse de manera clara en este estudio pues en cuanto a las características de la inserción laboral del grupo encuestado, el 90\% de los egresados labora en instituciones públicas, ya sea de asistencia o seguridad social, y en forma predominante en el 3er nivel de atención.

Respecto al ingreso salarial poco más del $70 \%$ de los egresados refiere que oscilan entre los 9 y 20 mil pesos. Las actividades profesionales señaladas como predominantes en este grupo de estudio son las de tipo asistencial -en congruencia con lo que la formación académica tiene como objetivo- siguiendo en orden de importancia las de carácter administrativo con un $21 \%$ del total de respuestas.

Con relación a la obtención del grado de especialista por los egresados (Tabla 1), poco menos de la mitad ya se ha graduado y al correlacionar este indicador con la categoría, es posible inferir que en los tabuladores institucionales, éste logro ya está reconocido pues el 68\% de estos ocupa actualmente una plaza como enfermera (o) especialista o desempeña funciones de mayor responsabilidad como son la supervisión o jefatura de servicio, que representa más del doble comparado con las (los) no graduadas (os).

Respecto a las especialidades que mejor se han posicionado son: la Enfermería Perinatal, Cardiovascular e Infantil, ya que son las que mayoritariamente cuentan con la categoría de enfermera especialista o superior (Tabla 2).

\section{Tabla 1. Categoría laboral según titulación en la especialidad}

\begin{tabular}{|c|c|c|c|c|c|c|}
\hline \multirow{3}{*}{ Categoría } & \multicolumn{4}{|c|}{ Titulado } & & \\
\hline & \multicolumn{2}{|c|}{ SI } & \multicolumn{2}{|c|}{ NO } & \multicolumn{2}{|c|}{ Total } \\
\hline & Frec & $\%$ & Frec & $\%$ & Frec & $\%$ \\
\hline $\begin{array}{l}\text { Auxiliar-General- } \\
\text { Licenciada }\end{array}$ & 55 & 28.5 & 139 & 68.1 & 194 & 48.9 \\
\hline $\begin{array}{c}\text { Especialista-Jefe- } \\
\text { Supervisora- } \\
\text { Académico }\end{array}$ & 138 & 71.5 & 65 & 31.9 & 203 & 51.1 \\
\hline Total & 193 & 100 & 204 & 100 & 397 & 100 \\
\hline
\end{tabular}

* 20 casos sin dato en categoría o titulación

Tabla 2. Categoría laboral según especialidad*

\begin{tabular}{|c|c|c|c|c|c|c|}
\hline \multirow[t]{2}{*}{ ESPECIALIDAD } & \multicolumn{2}{|c|}{$\begin{array}{c}\text { Enfermera } \\
\text { Auxiliar, } \\
\text { General, } \\
\text { Licenciatura }\end{array}$} & \multicolumn{2}{|c|}{$\begin{array}{c}\text { Especialista, } \\
\text { Jefe, } \\
\text { Supervisora, } \\
\text { Académicos }\end{array}$} & \multicolumn{2}{|c|}{ Total } \\
\hline & Frec & $\%$ & Frec & $\%$ & Frec & $\%$ \\
\hline Cardiovascular & 32 & 49.2 & 33 & 50.8 & 65 & 100 \\
\hline Perinatal & 14 & 33.3 & 28 & 66.7 & 42 & 100 \\
\hline Salud Pública & 10 & 52.6 & 9 & 47.4 & 19 & 100 \\
\hline Infantil & 50 & 50.5 & 49 & 49.5 & 99 & 100 \\
\hline Adulto EC & 64 & 56.6 & 49 & 43.4 & 113 & 100 \\
\hline Anciano & 3 & 23.1 & 10 & 76.9 & 13 & 100 \\
\hline Rehabilitación & 18 & 60.0 & 12 & 40.0 & 30 & 100 \\
\hline
\end{tabular}

"Se consideraron sólo las especialidades con mayor tiempo de establecidas 

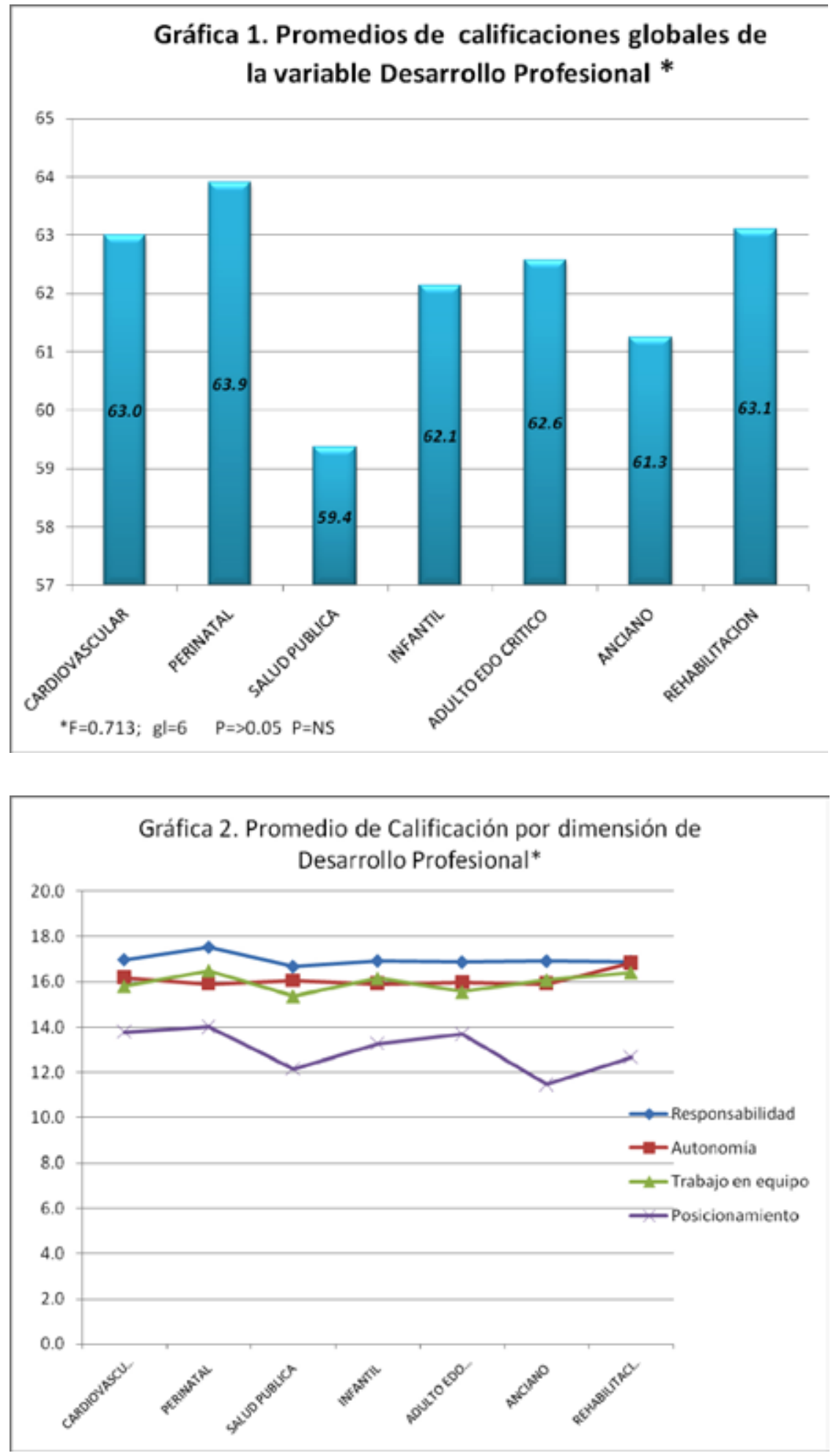

\begin{tabular}{rrrrr} 
& Responsabilidad & Autonomía & $\begin{array}{r}\text { Trab en } \\
\text { Equipo }\end{array}$ & Posicionamiento \\
\hline $\mathrm{X}^{2}=$ & 7.31 & 6.61 & 7.00 & 11.78 \\
$\mathrm{Gl}$ & 6 & 6 & 6 & 6 \\
Sig Asintót & 0.293 & 0.358 & 0.321 & 0.067
\end{tabular}

Al realizar el análisis específico respecto a la variable Desarrollo Profesional si se toma como indicador la calificación global obtenida a partir de la suma de las cuatro dimensiones de que constó la Escala (Gráfica 1), se tiene que la especialidad en que se obtuvo menor calificación fue en la de Salud Pública y en la del Anciano, sin embargo estas diferencias evaluadas a través de la prueba de ANOVA que permite estimar la variancia entre grupos por especialidad no son estadísticamente significativas. Con la misma prueba, se realizó un análisis al interior de cada grupo de especialidad para comparar las dimensiones de la variable Desarrollo Profesional (Gráfica 2), se observó que la dimensión de posicionamiento es la que alcanza una menor media en todos los casos. Sin embargo, no se obtiene un nivel de significancia estadística.

\section{Discusión y Conclusiones}

Los datos provenientes de este estudio reflejan que las características de la inserción laboral de los egresados del Plan Único de Especialización en Enfermería de la ENEO-UNAM es acorde a las expectativas del programa que lo sustenta puesto que en mayor medida este grupo de profesionales está ubicado en instituciones de 3er nivel, realizando actividades relacionadas con el cuidado especializado. Básicamente se logra la inserción en instituciones públicas que son las que tienen mejor delimitadas las diferenciaciones entre formación y posición en el empleo. Las remuneraciones también corresponden con lo esperado para profesionales con este nivel de formación ubicados en institucio- 
nes públicas. Sin embargo, un dato que debe considerarse para mejorar la posición de los egresados es la relación observada entre categoría en el empleo y el contar con el titulo de grado. Es decir, se deben impulsar acciones y contemplar mecanismos necesarios para mejorar la eficiencia terminal de sus egresados a fin de que estos tengan mayores posibilidades de ascenso a un puesto acorde a su formación.

Por otra parte, los resultados relacionados con las dimensiones contempladas para evaluar el desarrollo profesional (responsabilidad, autonomía, trabajo en equipo y posicionamiento) también aportan información valiosa para el PUEE, ya que de acuerdo con la percepción de los egresados la formación recibida durante la especialidad les brinda las habilidades necesarias para actuar con responsabilidad, autonomía y trabajar en equipo. Sin embargo, no les ha ayudado en igual medida para obtener el reconocimiento institucional o de colegas, o a mejorar su posición laboral, ni sus remuneraciones. Esto refleja que aún hay importantes tareas a realizar por las Asociaciones profesionales para lograr avances en el reconocimiento de la profesión, en el que es inherente la adecuada retribución salarial. Visto desde otro ángulo lo que se muestra también en este estudio es la racionalidad diferente en que se mueven el mercado laboral y las instituciones educativas respondiendo a las políticas públicas, unas buscando formar mejores profesionales universitarios que respondan a las necesidades de la sociedad y a las necesidades del sector salud formando los especialistas requeridos, pero el avance en la creación de plazas o puestos de trabajo acordes a esta formación no han ocurrido con la misma celeridad.

\section{Referencias Bibliográficas}

1 UNESCO 2000. Foro Mundial sobre la Educación. Marco de acción de Dakar. Educación para todos: cumplir nuestros compromisos comunes. Dakar, 26 al 28 de abril. París: UNESCO. Disponible en: http://bit.ly/xDQuk6 [Consultado 12 de marzo 2011]

2 México. Gobierno de la República. Plan Nacional de Desarrollo 20072012. pp. 12.

3 La Belle TJ. Educación no formal e informal: una perspectiva holística sobre el aprendizaje vitalicio. International Review of education 1982; 28(2): 159-75.

4. Salamón M. Panorama de las principales corrientes de interpretación de la Educación como fenómeno social. Perfiles Educativos CISEUNAM 1980,8:2-24.

5 Boff L. Prefacio. En: Assmann H. Reencantar a Educacao-rumo a sociedade aprendente. Petrópolis: Vozes; 1998. p 11.

6 ENEO. División de Estudios Profesionales. Plan de estudios de la Licenciatura en Enfermería y Obste- tricia. Perfil de egreso. Disponible en: http://bit.ly/xI3RGL

[Consultado 18 de mayo 2009]

7 Prado ML, Reibnitz KS. Salud y globalización: retos futuros para el cuidado de Enfermería. Invest Educ Enferm. 2004; 22(2): 104-11.

8 Oguisso T. A educacao continua como fator de mudancas: visao mundial. Rev Rec Enf Nursing. 2000; 20(1): 22-9.

9 Puntunet BM, Domínguez BA. La educación continua y la capacitación del profesional de enfermería. Revista Mexicana de Enfermería Cardiológica. 2008. 16(3): 115-17.

10 Arroyo de Cordero G. Actualización en Enfermería, una responsabilidad profesional. Rev Enferm IMSS. 2002; 10 (1): 3-5.

11 Chiodelli N, Do Prado ML. Educación continúa en el trabajo: Nueve desafíos para los profesionales de Enfermería. Investigación y Educación en Enfermería, 2007; XXV(1): 100-5.

12 ENEO. División de Estudios de Posgrado. Antecedentes y Perfil profesional de egresados del Plan Único de Especialidades de Enfermería (PUEE). Disponible En: http://bit. ly/ArOc4g [Consultado 10 de julio 2008]. 